by the statement that "Neuropsychological and neuroimaging studies have not shown robust differences between DLB and AD subjects". There is considerable evidence from published data to suggest that there is a clear difference in the neuropsychological profile between dementia with Lewy bodies (DLB) and Alzheimer's disease (AD) and that this could contribute to making the differential diagnosis.

The literature on the neuropsychological profile of DLB has been extensively reviewed by Salmon \& Galasko (1996). They summarise earlier work by Hansen's group which showed greater deficits in attention, verbal fluency and visuospatial processing in nine pathologically confirmed Lewy body variant patients compared with $10 \mathrm{AD}$ patients, as well as data from the Newcastle Group, using the CANTAB, a computerised battery of tests. In the latter study DLB patients were more impaired on delayed matching to sample, conditional learning paired associate tasks and spatial working memory. This difference in visuospatial ability was thought to reflect greater dysfunction of frontal lobe structures. The visuospatial differences have recently been shown to be of sufficient magnitude to be detected even by tests commonly used in clinical practice, e.g. on the CAMCOG (Walker et al, 1997a) and the Clock Face Test (Gnanalingham et al, 1997), suggesting those differences could be utilised to distinguish DLB from other dementias in a clinical setting.

As for neuroimaging studies, both perfusion studies (Albin et al, 1996) and dopamine receptor imaging (Walker et al, $1997 b$ ) have shown significant differences in the two conditions, suggesting that those might be a further aid to differentiate the two conditions.

Albin, R. L., Minoshima, M. D., D'Amato, B. S., et ol (1996) Fluorodeoxyglucose positron emission tomography in diffuse Lewy body disease. Neurology, 47, 462-466.

Gnanalingham, K. K., Byrne, E. J., Thornton, A., ot of (1997) Motor and cognitive function in Lewy body dementia: comparison with Alzheimer's and Parkinson's disease. Journal of Neurology. Neurosurgery and Psychiotry, 62. 243-252.

Miller, B. L. (1997) Clinical advances in degenerative dementias. British Journal of Psychiotry. 17I, 1-3.

Salmon, D. P. Galasko, D. (1996) Neuropsychological aspects of Lewy body dementia. In Dementio with Lewy Bodies (eds R. Perry, I. McKeith \& E. Perry). pp. 99-113. Cambridge: Cambridge University Press.

Walker, Z., Allen, R. L., Shergill, S. S., et of (19970) Neuropsychological performance in Lewy body dementia and Alzheimer's disease. British fournol of Psychiatry. 170. 156-158.
—, Costa, D. C., Jansen, A. G., et al (1997b) Dementia with Lewy bodies: a study of post synaptic dopaminergic receptors with iodine-123 iodobenzamide single-photon emission tomography. Europeon Journal of Nuclear Medicine. 24. 609-614.

R. L. Allen Essex and Herts Community NHS Trust Z. Walker, C. L. E. Katona Department of Psychiatry, University College London Medical School, Wolfson Building, Riding House Street, London WIN 8AA

\section{Tests of 'dissociation' and mood disorder}

Sir: Nijenhuis et al (1997) maintain that what Fahy (1988) and Merskey (1992) regard as the misdiagnosis of some cases of bipolar disorder as dissociative conditions (particularly multiple personality disorder) permits the hypothesis that, if we are right, instruments measuring dissociative pathology should give high scores, both in patients with supposed dissociative disorders and in those with bipolar affective disorder. Their claim is invalid. Patients with bipolar disorder may be misdiagnosed in at least two ways. First, as in the double consciousness cases, the existing natural phenomena are simply misread for honest, but antiquated reasons. Second, patients with bipolar disorder may be educated into producing the desired states that are to be labelled dissociative. It was not to be expected that patients with bipolar disorder would match the dissociative disorder group on these scales unless the former had been indoctrinated. Nijenhuis et al have compared un-indoctrinated subjects with others whom they consider to be dissociative and have obtained a number of highly significant statistical results which are predictable, but not for the reason they suppose. They comparison that has been offered is worthless.

Fahy, T. A. (1988) The diagnosis of multiple personality disorder. A critical review. British Journal of Psychiotry. 153. 597-606.

Merakey, H. (1992) The manufacture of personalities. The production of multiple personality disorder. British fournal of Psychiatry, 160, 327-340.

Nijenhuis, E. R. S., Spinhoven, P., Van Dyck, R., et of (1997) Dissociative pathology discriminates between bipolar mood disorder and dissociative disorder (letter). British Journal of Psychiatry, 170. 581.

H. Merskey London Psychiatric Hospital, University of Western Ontario. 850 Highbury Avenue, PO Box 2532, London, Ontario, Canada N6A 4HI

\section{Large same-year effects: fact or} artefact?

Sir: Kessler et al (1996) report inordinately large odds ratios for 'same-year' effects for major depressive disorder (MDD) and comorbid disorders (see Table 4). An examination of the estimation method indicates that the large odds are likely to be an artefact.

The authors used survival models to estimate time-lagged, same-year, and timetrend effects (Table 4). The three timedependent covariates were: (a) same-year $z_{1}=1$ if MDD and prior disorder occurred in the same year, 0 otherwise; (b) timelagged $-z_{2}=1$ if prior disorder occurred one or more years prior to onset of MDD, 0 otherwise; (c) time-trend $-x=$ number of years since onset of prior disorder.

The estimation of the odds ratio is similar to that of the Mantel-Haenszel approach with two-by-two tables defined at each event (MDD) time (Crowley, 1975, 1980). For $z_{1}$ this table would be:

\begin{tabular}{|c|c|c|c|}
\hline & \multicolumn{3}{|c|}{ MDD } \\
\hline & & Yes & No \\
\hline Other disorder and & Yes & A & B \\
\hline MDD in same year & No & C & D \\
\hline
\end{tabular}

By definition, cell $B$ in the table is always 0; no subject can be both 'no' for MDD and 'yes' for other disorder and MDD in the same year. This 'structural zero' inflates the odds ratio for the 'sameyear' effect, which could account for the high odds ratios in Table 4.

An approach that avoids this artefact (although still problematic, as indicated below) would be to include two binarytime dependent variables: $z_{3}=1$ if time since prior disorder is less than one year, 0 otherwise; $z_{4}=1$ if time since prior disorder is greater than one year, 0 otherwise.

Variable $z_{3}$, which measures within-year effect, does not result in a structural zero. A subject may have a prior disorder for less than a year but not have MDD when evaluated at another subject's event time. In other words, whereas $z_{1}$ requires that the onsets of the two disorders within an individual occur in the same year, $z_{3}$ does not.

To illustrate the two approaches, a simulation study was run. The generated data consisted of 1000 observations from an exponential distribution. The prevalence of MDD and prior disorder were set at $20 \%$. Distributions were chosen so that the MDD odds ratio for all years including 